\title{
Answer to the Letter to the Editor of I. Berg et al. concerning "Adolescent idiopathic scoliosis treated with posteromedial translation: radiologic evaluation with a 3D low-dose system" by B. Ilharreborde, G. Sebag, W. Skalli and K. Mazda; Eur Spine J (2013) Apr 12 [doi:10.1007/s00586-013-2902-6]
}

\section{B. Ilharreborde}

Received: 7 July 2013/Published online: 17 July 2013

(C) Springer-Verlag Berlin Heidelberg 2013

We thank I. Berg et al. for their interest in our article. We are aware that malocclusion features have been reported more frequently in AIS patients than in the control population, but although all our patients were seen by an experimented dentist before surgery in order to look for an infection site, we have not specifically studied their occlusal variables or their facial asymmetry. This topic might be of interest in the future and we completely agree that low-dose stereoradiography using EOS device represents a promising and very adapted tool to carry on such research projects. Since anteroposterior and lateral acquisitions of the skull are necessary to analyze the global spinal balance, future studies should focus on the development and validation of a 3D-specific model of the facial skeleton that could later be added in the reconstruction software and, therefore, be forwarded to the attending orthodontist.

Conflict of interest None.

B. Ilharreborde $(\bowtie)$

Pediatric Orthopaedic Department, Robert Debré Hospital,

48 Bd Sérurier, 75019 Paris, France

e-mail: i_brice@hotmail.com 